\title{
Oscillation of nonlinear differential-integral equation in a Banach space with respect to its subspace
}

N. A. Perestyuk and V. YU. Slusarchuk 


\title{
OSCILLATION OF NONLINEAR DIFFERENTIAL-INTEGRAL EQUATION IN A BANACH SPACE WITH RESPECT TO ITS SUBSPACE
}

\author{
N.A. PERESTYUK AND V.YU. SLUSARCHUK
}

[Received: January 17, 2003]

\begin{abstract}
The conditions for oscillation of the solution of a nonlinear differentialintegral equation in a Banach space with respect to its subspace are established.

Mathematics Subject Classification: 34C15, 34G20

Keywords: differential-integral equation, Banach space, oscillation with respect to a subspace, impulse system
\end{abstract}

\section{INTRODUCTION}

Let $E$ be a real Banach space and let $E_{1}$ be an arbitrary subspace of $E$ with $\operatorname{codim} E_{1}=1$. Let also $\varphi$ be a linear continuous functional on $E$ with $\operatorname{ker} \varphi=E_{1}$. Finally, let $P$ be a class of functions $p:[0,+\infty) \rightarrow R$, which are non-decreasing on $[0,+\infty)$ and left continuous on $(0,+\infty)$ and such that

$$
\lim _{t \rightarrow 0^{+}} p(t)=p(0) \text { and } \sup _{t \geq 0}(p(t+1)-p(t))<+\infty .
$$

Consider now the following differential-integral equation

$$
\frac{d_{-} x(t)}{d t}-\frac{d_{+} x(t)}{d t}+\sum_{k=1}^{n} \int_{s}^{t}\left[d p_{k}(u)\right] F_{k}(x(u))=0, t \geq s \geq 0,
$$

where: $n \in N ; p_{k} \in P, k=1,2, \ldots, n ; F_{k}: E \rightarrow E, k=1,2, \ldots, n$

are continuos mappings; the integrals in (1.1) are Lebesque-Stiltjes ones; $\frac{d_{-} x(t)}{d t}$ and $\frac{d_{+} x(t)}{d t}$ are the left and right derivatives of the solution $x(t)$ of (1.1) at the point $t$, respectively. Observe that at the point $t=0$ the left derivative of $x(t)$ is $\frac{d_{-} x(0)}{d t}$, which is compatible with the requirement $\lim _{t \rightarrow 0^{+}} p_{k}(t)=p_{k}(0), k=1,2, \ldots, n$, and the continuity of $F_{k}, k=1,2, \ldots, n$, on $E$.

As it is known (see, for example, [6]), each function $p_{k}(t), t \geq 0, k=1,2, \ldots, n$, can be represented in the form 


$$
p_{k}(t)=p_{c, k}(t)+p_{s, k}(t)+p_{d, k}(t), \quad k=1,2, \ldots, n,
$$

where $p_{c, k}(t)$ is an absolutely continuous function on each segment of the form $[0, a]$ with $a \in(0,+\infty)$, while $p_{s, k}(t)$ and $p_{d, k}(t)$ are the singular and discrete components of $p_{k}(t)$, respectively.

If in (1.3) for every $t \geq 0$

$$
p_{s, k}(t) \equiv \text { const }, \quad k=1,2, \ldots, n,
$$

then equation (1.1) is equivalent to a differential equation with impulse disturbance, while if in (1.3) for every $t \geq 0$

$$
p_{s, k}(t) \equiv \text { const } \text { and } p_{d, k}(t) \equiv \text { const }, \quad k=1,2, \ldots, n,
$$

then equation (1.1) is equivalent to a differential equation without impulse disturbance. Such differential equations have been studied in [3] and [8], when $\operatorname{dim} E<$ $+\infty$, and in [2]. Finally, note that if, in (1.3), for every $t \geq 0$,

$$
p_{c, k}(t) \equiv \text { const } \text { and } p_{s, k}(t)=\text { const }, \quad k=1,2, \ldots, n,
$$

then equation (1.1) is equivalent to a difference equation, the theory of which has been developed in [5].

The purpose of this article is to give conditions for oscillation of the solution of equation (1.1) with respect to the subspace $E_{1}$ of the Banach space $E$. The notion of such oscillation is stated and used successfully in [10] - [14] and [16] and is defined as follows.

Definition 1. A solution $x(t)$ of equation (1.1) is said to be oscillatory with respect to the subspace $E_{1}$ of the Banach space $E$, if for every number $a>0$ there are points $\tau_{1}, \tau_{2} \in(a,+\infty)$ such that

$$
\varphi\left(\tau_{1}\right) \varphi\left(\tau_{2}\right)<0 .
$$

As it follows from [10] - [14] and [16], this definition is a convenient tool for the investigation of oscillation of trajectories of dynamical systems with infinite dimensional phase space. Note that, in the case when $\operatorname{dim} E_{1}=1$, the above definition of the oscillation of a solution of (1.1) with respect to $E_{1}$ is equivalent to the corresponding one given the real solutions of the differential equations considered, for example, in [9].

An interesting class of equations is the class of differential equations with impulse disturbance, which so far has not been investigated sufficiently. Therefore, it is very important for the theory of systems with impulse, as well as for the theory of differential-integral equations to investigate the oscillatory nature of solutions of equations of form (1.1). 


\section{Preliminaries}

In order to state our main results, we need the following notions and assumptions.

(I) Assume that for any number $t_{0} \geq 0$ and for any set $\left\{x_{1}, x_{2}\right\} \subset E$ equation (1.1) admits a unique solution $x(t)$, which is defined and continuous on $[0,+\infty)$ and satisfies the following two conditions:

$$
x\left(t_{0}\right)=x_{1}
$$

and

$$
\frac{d_{+} x\left(t_{0}\right)}{d t}=x_{2}
$$

where, if $\left(x_{1}, x_{2}\right) \in E_{1}^{2}$, then $x(t) \in E_{1}$ for all $t \geq t_{0}$.

A solution of (1.1), satisfying (2.1) and (2.2), will be denoted by

$$
x\left(t, t_{0}, x_{1}, x_{2}\right) .
$$

For some sufficient conditions, concerning the requirement (I), see [17].

(II) Consider the sets

$$
E_{2}=\{x \in E: \varphi(x)>0\} \text { and } E_{3}=\{x \in E: \varphi(x)<0\},
$$

and the continuous mappings

$$
F_{k}: E \rightarrow E, k=1,2, \ldots, n .
$$

Assume that the sets $E_{1}, E_{2}$ and $E_{3}$ are invariant ones with respect to each of the mappings (2.3), i.e.,

$F_{k} E_{i} \subset E_{i}$ for all $k=1,2, \ldots, n$, and all $i=1,2,3$.

(III) Let $D_{k}$ be the set of all points of $[0,+\infty)$ in which the function $p_{k} \equiv p_{k}(t)$ is differentiable, and let $D$ be the intersection of the sets $D_{k}, k=1,2, \ldots, n$. Denote by $\Phi_{k}$ the set of functions $z \equiv z_{k}(t)$ which are continuous on $[0,+\infty)$ and differentiable on $D$ with values in $E_{i}, i=1,2,3$, for each of which

$$
\left|\varphi\left(z_{k}(t)\right)\right|, \quad k=1,2, \ldots, n,
$$

is a monotone non-decreasing on $[0,+\infty)$ function.

Note that, because of the inclusion $p \in P$, the set $[0,+\infty) / D_{k}$ has zero Lebesque measure.

\section{Main Results}

Here we formulate and prove a sufficient result (Theorem 1) and a necessary result (Theorem 2) as follows.

Theorem 1. Consider equation (1.1), subject to the conditions (I) - (III), and assume that for $k=1,2, \ldots, n$ :

(i) $\inf _{s \geq t \geq 0} \frac{\varphi\left(F_{k}(z(s))\right)}{\varphi\left(F_{k}(z(t))\right)}>0$ for all $z \in \Phi_{2} \cup \Phi_{3}$, 
(ii) The improper integrals

$$
\int_{0}^{+\infty} \frac{d \varphi(z(t))}{\varphi\left(F_{k}(z(t))\right)}
$$

converge for all $z \in \Phi_{2} \cup \Phi_{3}$ and

(iii) $\sum_{k=1}^{n} \int_{0}^{+\infty} t d p_{k}(t)=+\infty$.

Then all solutions $x\left(t, 0, x_{1}, x_{2}\right)$, where $\left(x_{1}, x_{2}\right) \in E^{2} \backslash E_{1}^{2}$, of equation (1.1) are oscillatory with respect to the subspace $E_{1}$.

Proof. Let $z(t)$ be non-oscillatory with respect to $E_{1}$ solution of (1.1), for which

$$
\left(z(0), \frac{d_{+} z(0)}{d t}\right) \notin E_{1}^{2} .
$$

Then, without loss of generality, we can (and do) suppose that for some $a \in(0,+\infty)$

$$
\varphi(z(a))>0 \text { and } \varphi(z(t)) \geq 0, \quad t \geq a .
$$

Since, in view of (1.1), for arbitrary $t \geq a$ and $s>t$

$$
\frac{d_{-} z(s)}{d s}-\frac{d_{+} z(t)}{d t}+\sum_{k=1}^{n} \int_{t}^{s}\left[d p_{k}(u)\right]=0
$$

and consequently for $s \geq t \geq 0$

$$
\varphi\left(\frac{d_{-} z(s)}{d s}\right)-\varphi\left(\frac{d_{+} z(t)}{d t}\right)+\sum_{k=1}^{n} \int_{t}^{s}\left[d p_{k}(u)\right] \varphi\left(F_{k}(z(u))\right)=0
$$

and, by (I) - (III) and (3.2), we have

$$
\sum_{k=1}^{n} \int_{t}^{s}\left[d p_{k}(u)\right] \varphi\left(F_{k}(z(u))\right) \geq 0
$$

for all $t$ and $s$ with $s>t \geq a$, we conclude that the function $\varphi\left(\frac{d_{+} z(t)}{d t}\right)$ is non-increasing on $[a,+\infty)$. Thus, the function $\varphi(z(t))$ is concave on $[a,+\infty)$ (see, [18], page 17) and, in view of (3.2) and the concavity of the function $\varphi(z(t))$ on $[a,+\infty)$, we derive that

$$
\lim _{t \rightarrow+\infty} \varphi\left(\frac{d_{+} z(t)}{d t}\right)=c, \quad c \in[0,+\infty) .
$$


Now, (3.4) and (3.3) imply that function $\varphi(z(t))$ is monotone non-decreasing on $[a,+\infty)$ and for all $t \geq a$

$$
\varphi\left(\frac{d_{+} z(t)}{d t}\right)=c+\sum_{k=1}^{n} \int_{t}^{+\infty}\left[d p_{k}(u)\right] \varphi\left(F_{k}(z(u))\right) .
$$

Denote by $\delta$ the least of the numbers

$$
\inf _{s \geq t \geq 0} \frac{\varphi\left(F_{k}(z(s))\right)}{\varphi\left(F_{k}(z(t))\right)}>0, k=1,2, \ldots, n \text { and } z \in \Phi_{2} \cup \Phi_{3}
$$

which, by (i), is positive. Taking into account the inclusion $z \in \Phi_{2}$, (ii) and (3.5), we see that

$$
\begin{aligned}
& +\infty>\int_{a}^{+\infty} \frac{d \varphi(z(t))}{\varphi\left(F_{k}(z(t))\right)}>\int_{a+1}^{+\infty} \frac{d \varphi\left(\frac{d_{+} z(t)}{d t}\right)}{\varphi\left(F_{k}(z(t))\right)}= \\
& =\int_{a+1}^{+\infty} \frac{1}{\varphi\left(F_{k}(z(t))\right)}\left(c+\sum_{k=1}^{n} \int_{t}^{+\infty}\left[d p_{k}(u)\right] \varphi\left(F_{k}(z(u))\right)\right) d t \geq \\
& \geq \int_{a+1}^{+\infty}\left(\int_{t}^{+\infty}\left[d p_{k}(u)\right] \frac{\varphi\left(F_{k}(z(u))\right)}{\varphi\left(F_{k}(z(t))\right)}\right) d t \geq \delta \int_{a+1}^{+\infty}(t-a-1) d p_{k}(t),
\end{aligned}
$$

which implies that the improper integrals

$$
\int_{a+1}^{+\infty} t d p_{k}(t), k=1,2, \ldots, n,
$$

are convergent. But this contradicts (iii). The contradiction obtained shows that the assumption (2.3) is false and the proof of the theorem is complete.

In order to state our next result, we need the following

Definition 2. A mapping $h: E \rightarrow E$ is called Lipschitzian, if for arbitrary $b \in E$ and $r \in(0,+\infty)$ there exists a constant $M>0$ such that

$$
\|h(x)-h(y)\|_{E} \leq M\|x-y\|_{E}
$$

for all $x, y \in B(b, r)=\left\{x \in E:\|x-b\|_{E} \leq r\right\}$.

Theorem 2. Consider equation (1.1), subject to the conditions (I) - (III), and assume that:

(iv) the mappings $F_{k}, k=1,2, \ldots, n$, are:

(a) locally Lipschitzian, or

(b) completely continuous;

(v) the functions $p_{k} \in P, k=1,2, \ldots, n$, are continuous on $[0,+\infty)$;

(vi) all solutions $x\left(t, 0, x_{1}, x_{2}\right)$, where $\left(x_{1}, x_{2}\right) \in E^{2} \backslash E_{1}^{2}$, of equation (1.1) are oscillatory with respect to $E$.

Then condition (iii) of Theorem 1 holds, i. e., 
(iii) $\sum_{k=1}^{n} \int_{0}^{+\infty} t d p_{k}(t)=+\infty$.

Proof. Case 1. The mappings $F_{k}, k=1,2, \ldots, n$, are locally Lipschitzian, i.e. (iv,a) holds. In this case, assume that (iii) is false, i.e.

$$
0 \leq \sum_{k=1}^{n} \int_{0}^{+\infty} t d p_{k}(t)<+\infty .
$$

For an arbitrary vector $y \in E_{2} \cup E_{3}$, and a closed ball $B(y, r), r>0$, for which $E_{1} \cap B(y, r)=\emptyset$, consider the equation

$$
z(t)=y-\sum_{k=1}^{n} \int_{t}^{+\infty}\left[d p_{k}(u)\right](u-t) F_{k}(z(u)), t \geq a,
$$

where $a$ is chosen in such a way that

$$
\sum_{k=1}^{n} \int_{a}^{+\infty}\left[d p_{k}(u)\right](u-a) \sup _{x \in B(y, r)}\left\|F_{k}(x)\right\|_{E} \leq r
$$

and

$$
\begin{aligned}
& \sup _{t \geq a}\left\|\sum_{k=1}^{n} \int_{t}^{+\infty}\left[d p_{k}(u)\right](u-t) F_{k}\left(z_{1}(u)\right)-\sum_{k=1}^{n} \int_{t}^{+\infty}\left[d p_{k}(u)\right](u-t) F_{k}\left(z_{2}(u)\right)\right\|_{E} \leq \\
& \leq \frac{1}{2} \sup _{t \geq a}\left\|z_{1}(t)-z_{2}(t)\right\|_{E}
\end{aligned}
$$

for all $E$-valued functions $z_{i}(t), i=1,2$ which are continuous and bounded on $[a,+\infty)$ for which

$$
\sup _{t \geq a}\left\|z_{i}(t)-y\right\|_{E} \leq r, \quad i=1,2 .
$$

Note that, because of (iv,a) and (3.3), the relations (3.8) and (3.9) are valid.

Next consider a Banach space $X$ of $E$-valued functions $x=x(t)$ which are continuous and bounded on $[a,+\infty)$ with the norm

$$
\|x\|_{E}=\sup _{t \geq a}\|x(t)\|_{E} .
$$

Furthermore, consider a bounded, closed and convex set $Y$ of all functions $x \in X$, such that $x \in B(y, r)$ for all $t \geq a$, and an operator $U: X \rightarrow X$ defined by the formula

$$
(U x)(t)=y-\sum_{k=1}^{n} \int_{t}^{+\infty}\left[d p_{k}(u)\right](u-t) F_{k}(x(u)), t \geq a
$$

Using (3.8) and (3.9), from (3.10) it follows that $U Y \subset X$ and $\|U v-U w\|_{X} \leq$ $\frac{1}{2}\|v-w\|_{X}$ for all $v, w \in Y$. Hence, applying the contraction mapping principle (see, 
[15], page 72), we conclude that there is a function $z \in Y$ which is a solution of equation (3.7). The same function $z$ is also a solution to equation (1.1). Indeed, by (3.6) and (v), we see that

$$
\begin{aligned}
& \frac{d-z(t)}{d t}=\lim _{\varepsilon \rightarrow 0} \frac{1}{\varepsilon}(z(t)-z(t-\varepsilon))=\lim _{\varepsilon \rightarrow 0} \frac{1}{\varepsilon}\left(-\sum_{k=1}^{n} \int_{t}^{+\infty}\left[d p_{k}(u)\right](u-t) F_{k}(z(u))+\right. \\
& \left.+\sum_{k=1}^{n} \int_{t-\varepsilon}^{+\infty}\left[d p_{k}(u)\right](u-t+\varepsilon) F_{k}(z(u))\right)= \\
& =\lim _{\varepsilon \rightarrow 0} \frac{1}{\varepsilon}\left(\varepsilon \sum_{k=1}^{n} \int_{t}^{+\infty}\left[d p_{k}(u)\right] F_{k}(z(u))+\sum_{k=1}^{n} \int_{t-\varepsilon}^{t}\left[d p_{k}(u)\right](u-t+\varepsilon) F_{k}(z(u))\right)= \\
& =\sum_{k=1}^{n} \int_{t}^{+\infty}\left[d p_{k}(u)\right] F_{k}(z(u))+\lim _{\varepsilon \rightarrow 0} \sum_{k=1}^{n} \int_{t-\varepsilon}^{t}\left[d p_{k}(u)\right] \frac{u-t}{\varepsilon} F_{k}(z(u))+ \\
& +\lim _{\varepsilon \rightarrow 0} \sum_{k=1}^{n} \int_{t-\varepsilon}^{t}\left[d p_{k}(u)\right] F_{k}(z(u)),
\end{aligned}
$$

where the two last terms tend to zero, because the integrand functions are bounded on $[t-\varepsilon, t], \underset{t-\varepsilon}{\stackrel{t}{\vee}}\left[p_{k}\right]=p_{i}, \underset{t-\varepsilon}{\vee}\left[p_{k}\right]=p_{k}(t)-p_{k}(t-\varepsilon), k=1,2, \ldots, n$, and the functions $p_{k}(t), k=1,2, \ldots, n$ are continuous on $[0,+\infty)$. Therefore, it follows that

$$
\frac{d_{-z}(t)}{d t}=\sum_{k=1}^{n} \int_{t}^{+\infty}\left[d p_{k}(u)\right] F_{k}(z(u)) .
$$

In the same manner we see that

$$
\begin{aligned}
& \frac{d_{+} z(s)}{d t}=\lim _{\varepsilon \rightarrow 0} \frac{1}{\varepsilon}(z(s+\varepsilon)-z(s))=\lim _{\varepsilon \rightarrow 0} \frac{1}{\varepsilon}\left(-\sum_{k=1}^{n} \int_{s+\varepsilon}^{+\infty}\left[d p_{k}(u)\right](u-s-\varepsilon) F_{k}(z(u))+\right. \\
& \left.+\sum_{k=1}^{n} \int_{s}^{+\infty}\left[d p_{k}(u)\right](u-s) F_{k}(z(u))\right)=\lim _{\varepsilon \rightarrow 0} \frac{1}{\varepsilon}\left(\varepsilon \sum_{k=1}^{n} \int_{s}^{+\infty}\left[d p_{k}(u)\right] F_{k}(z(u))+\right. \\
& \left.+\sum_{k=1}^{n} \int_{s}^{s+\varepsilon}\left[d p_{k}(u)\right] F_{k}(z(u))\right)= \\
& =\sum_{k=1}^{n} \int_{s}^{+\infty}\left[d p_{k}(u)\right] F_{k}(z(u))+\lim _{\varepsilon \rightarrow 0} \sum_{k=1}^{n} \int_{s}^{s+\varepsilon}\left[d p_{k}(u)\right] \frac{u-s}{\varepsilon} F_{k}(z(u))- \\
& -\lim _{\varepsilon \rightarrow 0} \sum_{k=1}^{n} \int_{s}^{s+\varepsilon}\left[d p_{k}(u)\right] F_{k}(z(u))=\sum_{k=1}^{n} \int_{s}^{+\infty}\left[d p_{k}(u)\right] F_{k}(z(u)) .
\end{aligned}
$$

Subtracting (3.12) from (3.11), we obtain

$$
\begin{aligned}
& \frac{d_{-} z(t)}{d t}-\frac{d_{+} z(s)}{d s}=\sum_{k=1}^{n} \int_{t}^{+\infty}\left[d p_{k}(u)\right] F_{k}(z(u))- \\
& -\sum_{k=1}^{n} \int_{s}^{+\infty}\left[d p_{k}(u)\right] F_{k}(z(u))=-\sum_{k=1}^{n} \int_{s}^{t}\left[d p_{k}(u)\right] F_{k}(z(u)),
\end{aligned}
$$


which was to be shown. As an element of $Y$, the function $z(t)$ is not oscillatory with respect to $E_{1}$. By (I) - (III), there is a solution $y(t)$ of equation (1.1), which coincides with $z(t)$ on $[a,+\infty)$. For this solution it holds

$$
\left(y(0), \frac{d_{+} y(0)}{d t}\right) \in E^{2} \backslash E_{1}^{2}
$$

and, due to the uniqueness of a solution $x(t)$ of (1.1), we see that

Note that, if

$$
x(0)=y(0) \text { and } \frac{d_{+} x(0)}{d t}=\frac{d_{+} y(0)}{d t} .
$$

$$
\left(x(0), \frac{d_{+} x(0)}{d t}\right) \in E_{1}^{2},
$$

then equation (1.1) has a solution $x(t)$ for which

$$
\left(x(0), \frac{d_{-} x(0)}{d t}\right) \in E_{1}^{2} \text { for all } t \geq 0 .
$$

From the above observation it follows that, if (iii) does not hold, then equation (1.1) has a non-oscillatory solution $x=x(t)$ with respect to $E_{1}$ for which

$$
\left(x(0), \frac{d_{+} x(0)}{d t}\right) \in E^{2} \backslash E_{1}^{2} \text {. }
$$

But this is a contradiction, which, in the case when the mapping $F_{k}, k=1,2, \ldots, n$ are locally Lipschitzian, proves the necessity of the condition (iii) for the oscillation of solution of (1.1) with respect to $E_{1}$.

Case 2. The mappings $F_{k}, k=1,2, \ldots, n$, are completely continuous, i.e., (iv, b) holds.

Assume that (iii) does not hold. Then (3.6) is satisfied. Let $y \in E_{2} \cup E_{3}$ be an arbitrary vector and let $r>0$ be a number such that $E_{1} \cap B(y, r)=\emptyset$. Next, consider the equation (3.7), where $a \in[0,+\infty)$ is such that (3.8) holds. Such a choice of $a$ is possible because of (3.6) and the compactness of the mappings $F_{k}, k=1,2, \ldots, n$. Further, as in Case 1, consider the Banach space $X$, the bounded, closed and convex set $Y$ of all functions $x \in X$ for which $x(t) \in B(y, r)$ for all $t \geq a$, and the operator $U: X \rightarrow X$ defined by the formula (3.10). By (3.10) and (3.8), it follows that $U Y \subset X$.

Consider now the function

$$
\delta(t)=L \sum_{k=1}^{n} \int_{t}^{+\infty}(u-t) d p_{k}(u), t \geq a,
$$

where

$$
L=\max _{\substack{1 \leq k \leq n \\ x \in B(y, r)}}\left\|F_{k}(x)\right\|_{E} .
$$


For arbitrary $y \in Y$ and $t, s \in[a,+\infty), t \geq s$, we see that

$$
\begin{aligned}
& \|(U y)(t)-(U y)(s)\|_{E}= \\
& =\left\|\sum_{k=1}^{n} \int_{t}^{+\infty}\left[d p_{k}(u)\right](u-t) F_{k}(y(u))-\sum_{k=1}^{n} \int_{s}^{+\infty}\left[d p_{k}(u)\right](u-s) F_{k}(y(u))\right\|_{E}= \\
& =\left\|\sum_{k=1}^{n} \int_{s}^{t}\left[d p_{k}(u)\right](u-t) F_{k}(y(u))-(t-s) \sum_{k=1}^{n} \int_{t}^{+\infty}\left[d p_{k}(u)\right] F_{k}(y(u))\right\|_{E} \leq \\
& \leq L(t-s) \sum_{k=1}^{n} \frac{t}{V}\left[p_{k}\right]+L(t-s) \delta(t) .
\end{aligned}
$$

Hence, in view of the fact that

$$
\sup _{\substack{1 \leq k \leq n \\ t \geq 0}} \underset{t}{V}\left[p_{k}\right]<+\infty,
$$

and because of the boundedness of the function $\delta(t)$ on $[a,+\infty)$, we conclude that the set of all $z \in U Y$ is equicontinuous on $[a,+\infty)$. Since for every $z \in U Y$

$$
\|z(t)-y\|_{E} \leq \delta(t), t \geq a
$$

and

$$
\lim _{t \rightarrow+\infty} \delta(t)=0
$$

in view of (i) and the generalized Arcela theorem (see, [4], page 110), we conclude that the set $U Y$ is relatively compact. Thus, $U: Y \rightarrow Y$ is a completely continuous mapping. Consequently, according to Schauder's Fixed Point Theorem (see, [7], p. 37), the mapping $U$ has a fixed point $z \in Y$, which is a solution of the equation $(1.1)$ on $[a,+\infty)$.

Finally, using arguments similar to those in Case 1, we come again to a contradiction. Therefore, the necessity of the condition (iii) for the oscillation of the solution of (1.1) with respect to $E_{1}$ in the case of compact mapping $F_{k}, k=1,2, \ldots, n$, is also established.

The proof of the theorem is complete.

\section{Applications}

Let $T$ be an arbitrary countable set of real numbers $t_{n}, n \in N$ with

$$
0<t_{1}<t_{2}<\ldots<t_{n}<\ldots \text { and } \lim _{n \rightarrow+\infty} t_{n}=+\infty .
$$

Moreover, let for $m \in N$

$$
c_{k}:[0,+\infty) \backslash T \rightarrow[0,+\infty), k=1,2, \ldots, m
$$

be bounded and continuous functions,

$$
q_{k}: T \rightarrow[0,+\infty), k=m+1, m+2, \ldots, 2 m,
$$


be arbitrary mappings with a bounded set of values, and

$$
G_{k}: E \rightarrow E, \quad k=1,2, \ldots, 2 m,
$$

be continuous mappings, for which

$$
G_{k} E_{i} \subset E_{i} \text { for all } k=1,2, \ldots, 2 m \text { and } i=1,2,3 .
$$

Then, consider an impulse system, described by the equations

$$
\left\{\begin{array}{l}
\frac{d^{2} x(t)}{d t^{2}}+\sum_{k=1}^{m} c_{k}(t) G_{k}(x(t))=0, \quad t \in[0,+\infty) \backslash T, \\
\frac{d_{+} x(t)}{d t}-\frac{d_{-} x(t)}{d t}+\sum_{k=m+1}^{2 m} q_{k}(t) G_{k}(x(t-0))=0, \quad t \in T, \\
x(t+0)=x(t-0)=x(t), \quad t \in T .
\end{array}\right.
$$

Furthermore, let

$$
p_{k}=p_{k}(t) \in P, \quad k=1,2, \ldots, 2 m,
$$

be functions defined as follows

$$
p_{k}(t)=\left\{\begin{array}{l}
\int_{0}^{t} c_{k}(s) d s \quad \text { for } k=1,2, \ldots, m, \\
\sum_{s \in[0, t) \cap T} q_{k}(s) \text { for } k=m+1, m+2, \ldots, 2 m,
\end{array}\right.
$$

where $\int_{0}^{t} C_{k}(s) d s$ is a Lebesque integral, while

$$
p_{k}(t)=0, \quad k=m+1, m+2, \ldots, 2 m, \quad \text { if } \quad[0, t) \cap T=\emptyset,
$$

and consider the equation

$$
\frac{d_{-} x(t)}{d t}-\frac{d_{+} x(t)}{d t}+\sum_{k=1}^{n} \int_{s}^{1}\left[d p_{k}(u)\right] G_{k}(x(u)), \quad t \geq s \geq 0 .
$$

It is not difficult to see that every solution of system (4.1) is a solution of equation (4.2) and vice versa. Therefore, Theorem 1 can be used to investigate the oscillation of solutions of system (4.1). As an application of Theorem 1 to system (4.1), we state the following result.

Theorem 3. Consider system (4.1) and assume that for arbitrary elements $x_{1}, x_{2} \in E$ system (4.1) has a unique solution $x\left(t, 0, x_{1}, x_{2}\right)$ defined on $[0,+\infty)$;

$$
\inf _{s \geq t \geq 0} \frac{\varphi\left(G_{k}(z(s))\right)}{\varphi\left(G_{k}(z(t))\right)}>0, \quad k=1,2, \ldots, 2 m,
$$


for all $z \in \Phi_{2} \cup \Phi_{3}$;

the improper integrals

$$
\int_{0}^{+\infty} \frac{d \varphi(z(t))}{\varphi\left(G_{k}(z(t))\right)}, k=1,2, \ldots, 2 m,
$$

converge for all $z \in \Phi_{2} \cup \Phi_{3}$, and

$$
\int_{0}^{+\infty} t \sum_{k=1}^{m} c_{k}(t) d t+\sum_{t \in T} t \sum_{k=m+1}^{2 m} q_{k}(t)=+\infty .
$$

Then for arbitrary $\left(x_{1}, x_{2}\right) \in E^{2} \backslash E_{1}^{2}$ solution $x\left(t, 0, x_{1}, x_{2}\right)$ of system (4.1) oscillates with respect to $E_{1}$.

Remark 1. 1. For sufficient conditions, ensuring the fulfillment of the conditions (B) and (C) of Theorem 3, see [16].

2. As it is shown in [16], in the case of locally Lipschitzian or completely continuous mappings $G_{k}, k=1,2, \ldots, 2 m$, the fulfillment of the condition $(D)$ is also a sufficient condition for oscillation of solutions $x\left(t, 0, x_{1}, x_{2}\right)$, with $\left(x_{1}, x_{2}\right) \in E^{2} \backslash E_{1}^{2}$, of system (4.1) with respect to $E_{1}$.

3. Necessary and sufficient conditions for oscillation of system (4.1), when $m=$ 1, $\operatorname{dim} E=1$ and condition $(C)$ is not satisfied, can be found in [15].

A special case of Theorems 1 and 2 is the following result, due to F. Atkinson [1]:

Theorem 4. Let $p(t)$ be a continuous and positive function for $t \geq 0$, and let $m$ be an integer greater than 1 . Then the condition

$$
\int_{0}^{+\infty} t p(t) d t=+\infty
$$

is a necessary and sufficient one for oscillation of all (except trivial) solutions of the equation

$$
y^{\prime \prime}(t)+p(t) y^{2 m-1}(t)=0 .
$$

\section{REFERENCES}

[1] Aткinson, F.: On second order non-linear oscillations,Pacific J. Math., 5, (1995), 643-647.

[2] Daletskit, Yu. L. and Kreyn M. G.: Stability of Solutions of Differential Equations in a Banach Space, Moscow, 1970. (in Russian)

[3] Halanay, A. and Wexler, D.: Qualitative Theory of Sampled-data Systems, Edita Acad. RSR, Bucharest, 1968 (in Rumanian). Russian edition in 1971 by "Mir", Moscow.

[4] Kolmogorov, A. N. and Fomin, S. V.: Elements of Theory of Functions and Functional Analysis, Moscow, 1968. (in Russian)

[5] Martynyuk, D. I.: Lectures on Qualitative Theory of Difference Equations, Kiev, 1972. (in Russian) 
[6] Natanson, I. P.: Theory of Real-variable Functions, Moscow, 1974. (in Russian)

[7] Nirenberg, L.: Lectures on Nonlinear Functional Analysis, Moscow, 1977. (in Russian)

[8] Samoylenko, A. M. and Perestyuk, N. A.: Differential Equations with Impulse Disturbance, Kiev, 1987. (in Russian)

[9] Shevelo, V. N.: Oscillation of Solutions of Differential Equations with a Deviating Argument, Kiev, 1978. (in Russian)

[10] Slusarchuk, V. Yu.: Oscillation of Solutions of Differential-difference Equations in a Banach Space (Ukrainian), "Nonlinear Differential Equations and their Applications", Kiev, 1993, pp. 66-70. (in Ukrainian)

[11] Slusarchuk, V. Yu.: Oscillation of solutions of differential equations with impulse disturbance in a Banach space, "Constructive methods of investigation of differential equations", Kiev, 1993, pp. 174-178. (in Ukrainian)

[12] Slusarchuk, V.Yu.: Sufficient conditions for oscillation of trajectories of impulse systems with unfixed moments of impulse disturbance), "Integral transformations and their applications to boundary-value problems", Kiev, 1994, pp. 192-197. (in Ukrainian)

[13] Slusarchuk, V.Yu.: Oscillation of solutions of the differential equation $\Delta^{2} x(n)+$ $\sum_{k=1}^{m} p_{k}(n) q_{k}(x(n))=0$ in a Banach space, In: Systems of Evolutionary Equations with Aftereffect, Institute of Mathematics, NAS of Ukraine, Kiev, 1995, pp. 98-102. (in Ukrainian)

[14] Slusarchuk, V. Yu.: Oscillation of solutions of difference equations in a Banach space, Proc. of Int. Math. Conference dedicated to the memory of Hans Han, Chernivtsi, 1995, pp. 169-175. (in Ukrainian)

[15] Slusarchuk, V. Yu.: Necessary and sufficient conditions for oscillation of solutions of nonlinear impulse system with multiplicatively divided right-hand part, Ukrain. Math. J., 47, (1995), 381398. (in Ukrainian)

[16] Slusarchuk, V. Yu.: Necessary and sufficient conditions for oscillation of solutions of nonlinear differential equations with impulse disturbance in a Banach space, Ukrain. Math. J., 51, (1999), 98-109. (in Russian)

[17] Slusarchuk, V. Yu.: General theorems for existence and uniqueness of solutions of differential equations with impulse disturbance (Russian), Ukrain. Math. J., 52 (2000), 954-964. (in Russian)

[18] Ushakov, R. R. and Hacet, B. I.: Convex Functions and Inequalities, Kiev, 1986. (in Ukrainian)

\section{Authors' Addresses}

\section{N.A. Perestyuk:}

Mechanical and Mathematical Faculty, Kyiv National Taras Shevchenko University, Volodymyrska, 64, 01033, Kyiv, UkRaine,

E-mail address: pmo@mechmat.univ.kiev.ua

\section{V.Yu. Slusarchuk:}

Current address: Mechanical and Mathematical Faculty, Kyiv National Taras Shevchenko University, Volodymyrska, 64, 01033, Kyiv, Ukraine,

E-mail address: pmo@mechmat.univ.kiev.ua 